\title{
From spatially indirect to momentum-space indirect exciton by in-plane magnetic field
}

\author{
L. V. Butov ${ }^{1}$, A. V. Mintsev ${ }^{1}$, Yu.E. Lozovik ${ }^{2}$, K. L. Campman ${ }^{3}$, and A. C. Gossard ${ }^{3}$ \\ ${ }^{1}$ Institute of Solid State Physics, Russian Academy of Sciences, 142432 Chernogolovka, Russia \\ ${ }^{2}$ Institute of Spectroscopy, Russian Academy of Sciences, 142092, Troitsk, Russia \\ ${ }^{3}$ Department of Electrical and Computer Engineering and Center for Quantized Electronic Structures (QUEST), University of \\ California, Santa Barbara, CA 93106
}

(December 11, 1999)

\begin{abstract}
In-plane magnetic field is found to change drastically the photoluminescence spectra and kinetics of interwell excitons in $\mathrm{GaAs} / \mathrm{Al}_{x} \mathrm{Ga}_{1-x} \mathrm{As}$ coupled quantum wells. The effect is due to the in-plane magnetic field induced displacement of the interwell exciton dispersion in a momentum space, which results in the transition from the momentum-space direct exciton ground state to the momentumspace indirect exciton ground state. In-plane magnetic field is, therefore, an effective tool for the exciton dispersion engineering.
\end{abstract}

The effective exciton temperature in a quasiequilibrium system of excitons in semiconductors is determined by the ratio between the exciton energy relaxation rate and the exciton recombination rate. The low exciton temperature is crucial for an observation of a number of novel collective phenomena caused by the high occupation of the lowest energy exciton states in a quasi-twodimensional (2D) exciton system [1 5]. Low temperatures can be achieved in a system with a low exciton recombination rate.

A long exciton lifetime is characteristic (1) for the systems where the ground state exciton is optically inactive (in dipole approximation) because of parity, e.g. for $\mathrm{Cu}_{2} \mathrm{O} ;(2)$ for the systems where electron and hole are spatially separated, e.g. for spatially indirect (interwell) excitons in direct-band-gap coupled quantum wells (CQWs) like $\Gamma-X_{z}$ AlAs/GaAs CQWs and GaAs/ $\mathrm{Al}_{x} \mathrm{Ga}_{1-x} \mathrm{As}$ CQWs; (3) for the systems where the ground state exciton is indirect in a momentum space, e.g. for $\Gamma-X_{x y} \mathrm{AlAs} / \mathrm{GaAs}$ CQWs.

Due to the coupling between the internal structure of magnetoexciton and its center-of-mass motion [6] the ground exciton state in a direct-band-gap semiconductor in crossed electric and magnetic fields was predicted to be at finite momentum. In particular, the transition from the momentum-space direct exciton ground state to the momentum-space indirect exciton ground state was predicted (1) for interwell exciton in coupled quantum wells in in-plane magnetic field [1, 7], and (2) for single layer exciton in in-plane electric field and perpendicular magnetic field 8 10]. These effects should allow for a controllable variation of the exciton dispersion and increase of the exciton ground state lifetime.

In the present paper we report on the experimental observation of the in-plane magnetic field induced transition from the momentum-space direct exciton ground state to the momentum-space indirect exciton ground state for the system of interwell excitons in $\mathrm{GaAs} / \mathrm{Al}_{x} \mathrm{Ga}_{1-x} \mathrm{As}$ CQWs. The transition is identified by the drastic change of the exciton photoluminescence (PL) kinetics. The in- terwell exciton dispersion in in-plane magnetic field is analyzed theoretically and determined experimentally from the shift of the interwell exciton PL energy; the experimental data are found to be in a qualitative agreement with the theory.

We suppose that the in-plane magnetic field is directed along the $x$-axis, and use the calibration $A_{x}=A_{z}=$ $0, A_{y}=-B z$. Due to the invariance corresponding to the simultaneous magnetic translation of electron and hole on the same vector parallel to the CQW plane, i.e. the invariance to the translation and corresponding gauge transformation, the two-dimensional magnetic momentum is conserved [6]. For the gauge used

$$
P_{x}=p_{e x}+p_{h x} ; P_{y}=p_{e y}+p_{h y}-\frac{e}{c} B\left(z_{e}-z_{h}\right) .
$$

For $z_{e, h}^{\prime}$ measured from the centers of the corresponding QW's one obtains $P_{y}=p_{e y}+p_{h y}-\frac{e}{c} B d-\frac{e}{c} B\left(z_{e}^{\prime}-z_{h}^{\prime}\right)$, where $d$ is the mean separation between the electron and hole layers. The value $p_{B}=-\frac{e}{c} B d=\hbar d / l_{B}^{2}$ is the shift of the magnetic momentum of interwell exciton in the ground state (as follows from the analysis of Shrodinger equation); $l_{B}=\left(\frac{e B}{\hbar c}\right)^{-\frac{1}{2}}$ is the magnetic length.

The physical sense of this shift can be obtained from the analysis of the adiabatic turning on of the in-plane magnetic field. The appearance of the vortex electric field leads to the acceleration of interwell exciton. The final in-plane momenta is equal precisely to $-\frac{e}{c} B d$ and directed normal to the magnetic field. Therefore, the appearance of the momentum $-\frac{e}{c} B d$ is related to the diamagnetic response of electron-hole (exciton) system in CQW in in-plane magnetic field.

The contribution of the second order on the magnetic field consist of two parts: the first, depending on the momenta, is the sum of Van Vleck paramagnetism of isolated QWs, and the second is the sum of diamagnetic shifts of isolated QWs [11]. Van Vleck paramagnetism leads to the renormalization of the effective (magnetic) mass of exciton along the $y$-axis, $M_{y y}$. Therefore, the magnetoexciton dispersion law becomes anisotropic: 


$$
M_{x x}=M=m_{e}+m_{h} ; M_{y y}=M+\delta M(B),
$$

where

$$
\begin{gathered}
\delta M(B)=\frac{e^{2} B^{2}}{c^{2}}\left(f_{e}+f_{h}\right), \\
-f_{e, h}=\sum_{n} \frac{|<0| z_{e, h}|n>|^{2}}{E_{0}-E_{n}} .
\end{gathered}
$$

Here $E_{n}, \mid n>$ are the energies and state vectors corresponding to the size quantization of QW's; $f_{e, h}$ are related to the electric polarization of QW's in the $z$ direction. $\delta M(B)>0$. The estimate results to:

$$
\frac{\delta M_{y y}}{M} \sim \frac{E_{\text {diam }}}{E} \sim\left(\frac{L_{z}}{l_{B}}\right)^{4}
$$

where $E_{\text {diam }}$ is the diamagnetic shift in isolated QW, $E$ is the size quantized excitation energy of QW, $L_{z}$ is the QW width.

The electric-field-tunable $n^{+}-i-n^{+}$GaAs/AlGaAs CQW structure was grown by MBE. The $i$-region consists of two $8 \mathrm{~nm}$ GaAs QWs separated by a $4 \mathrm{~nm}$ $\mathrm{Al}_{0.33} \mathrm{Ga}_{0.67} \mathrm{As}$ barrier and surrounded by two $200 \mathrm{~nm}$ $\mathrm{Al}_{0.33} \mathrm{Ga}_{0.67} \mathrm{As}$ barrier layers. The $n^{+}$-layers are Si-doped GaAs with $N_{\mathrm{Si}}=5 \times 10^{17} \mathrm{~cm}^{-3}$. The electric field in the z-direction is monitored by the external gate voltage $V_{g}$ applied between $n^{+}$-layers. Carriers were photoexcited by a pulsed semiconductor laser $(\hbar \omega=1.85 \mathrm{eV}$, the pulse duration was about $50 \mathrm{~ns}$, the edge sharpness including the system resolution was $\approx 0.5 \mathrm{~ns}$, the repetition frequency was $\left.1 \mathrm{MHz}, W_{e x}=10 \mathrm{~W} / \mathrm{cm}^{2}\right)$. In order to minimize the effect of the mesa heating, we worked with a mesa area of $0.2 \times 0.2 \mathrm{~mm}^{2}$, which was much smaller than the sample area of about $4 \mathrm{~mm}^{2}$. In addition, the bottom of the sample was soldered to a metal plate. The PL measurements were performed in a He cryostat by means of an optical fiber with diameter $0.1 \mathrm{~mm}$ positioned 0.3 $\mathrm{mm}$ above the mesa. The PL spectra and kinetics were measured by a time correlated photon counting system.

The separation of electrons and holes in different QWs (the indirect regime) is realized by applying a finite gate voltage which fixes external electric field in the $z$ direction $F=V_{g} / d_{0}$, where $d_{0}$ is the $i$-layer width [12]. The spatially direct (intrawell) and indirect (interwell) transitions are identified by the PL kinetics and gate voltage dependence: the intrawell PL line has short PL decay time and its position practically does not depend on $V_{g}$, while interwell PL line has long PL decay time and shifts to lower energies with increasing $V_{g}$ (the shift magnitude is given by $e F d$ ) 12, 13, . The upper and the lower direct transitions are related to the intrawell 1s heavy hole exciton $X$ and intrawell charged complexes $X^{+}$and $X^{-}$ [14.
With increasing in-plane magnetic field the energy of interwell exciton increases while the energies of the direct transitions are almost unaffected (Fig. 1a). This behaviour is consistent with a displacement of the interwell exciton dispersion in in-plane magnetic field. The scheme of the interwell exciton dispersion at zero and finite in-plane magnetic field is shown in Fig. 2.

For delocalized 2D excitons only the states with small center-of-mass momenta $k \leq k_{0} \approx E_{g} / \hbar c$ (where $c$ is the speed of light in the medium), i.e. within the radiative zone, can decay radiatively 15] (see Fig. 2). For GaAs structures $k_{0} \approx 3 \times 10^{5} \mathrm{~cm}^{-1}$ and is much smaller than $k_{B}=p_{B} / \hbar$ in strong fields (at $B=10 \mathrm{~T} k_{B} \approx 2 \times 10^{6}$ $\mathrm{cm}^{-1}$ ). The energy of the interwell exciton PL is set by the energy of the radiative zone. Therefore, as the diamagnetic shift of the bottom of the subbands is small and can be neglected to the first approximation, the interwell exciton PL energy in in-plane magnetic field should be increased by $E_{p=0}=p_{B}^{2} / 2 M=e^{2} d^{2} B^{2} / 2 M c^{2}$. In particular, the in-plane magnetic field dependence of the interwell PL energy could be used for the measurement of the exciton dispersion because it allows to access high momentum exciton states. At small fields the measured PL energy shift rate is $0.062 \mathrm{meV} / \mathrm{T}^{2}$ (see inset to Fig. 1a) which corresponds to $M=0.21 m_{0}$. This value is close to the calculated mass of heavy hole exciton in GaAs QWs $\approx 0.25 m_{0}\left(m_{e}=0.067 m_{0}\right.$ and the calculated in-plane heavy hole mass near $k=0$ is reported to be $m_{h}=0.18 m_{0}$, see Ref. [16] and references therein). Figure 1 shows considerable deviation from the quadratic dependence of the interwell exciton PL energy at high $B$. This deviation is the consequence of the interwell exciton mass renormalization due to in-plane magnetic field which is predicted by theory, see Eqs. 2-4. Due to the estimate of Eq. 5 the deviation of the interwell exciton dispersion from the quadratic one should become essential in the magnetic field where $l_{B} \sim L_{z}$. This is in a good qualitative agreement with the experiment which presents the onset of the deviation at $B \approx 8 \mathrm{~T}$, where $l_{B}=9 \mathrm{~nm} \sim L_{z}=8 \mathrm{~nm}$ (see inset to Fig. 1a). A possible contribution from the hole dispersion nonparabolicity to the observed increase of the exciton mass is, apparently, a minor effect for the small exciton energies considered which are much smaller than the light- heavy-hole splitting equal to $17 \mathrm{meV}$ for the CQW studied [14].

Note that the development of indirect gap when an in-plane magnetic field is applied has been observed in asymmetric modulation doped single quantum well where the centers of the electron and hole envelopes do not coincide; due to the free carrier character of recombination in the studied 2D electron gas, the PL energy shift corresponded to the electron mass [17].

In-plane magnetic field modifies qualitatively the exciton PL kinetics (Fig. 1b). The main feature of the interwell exciton PL kinetics at zero magnetic field is a sharp enhancement of the PL intensity after the exci- 
tation is switched off - the PL-jump 13] (Fig. 1). The basis of the effect is the following. The exciton PL kinetics is determined by the kinetics of occupation of the radiative zone (marked bold in Fig. 2). The occupation varies due to the exciton recombination and energy relaxation. The PL-jump denotes a sharp increase of the occupation of the optically active exciton states just after the excitation is switched off. It is induced by the sharp reduction of the effective exciton temperature, $T_{\text {eff }}$, due to the fast decay of the nonequilibrium phonon occupation and energy relaxation of hot photoexcited excitons, electrons, and holes [13]. The disappearance of the PLjump at high in-plane magnetic fields is consistent with the displacement of the interwell exciton dispersion in parallel magnetic field: for momentum-space indirect exciton a sharp reduction of $T_{\text {eff }}$ just after the excitation is switched off should not result in the increase of occupation of the radiative zone (see Fig. 2c) and, hence, the PL intensity.

The measured radiative decay rate is proportional to the fraction of excitons in the radiative zone. The observed strong reduction of the radiative decay rate in inplane magnetic field (by more than 20 times in $B=12$ T, see Fig. 1b) also reflects the displacement of the interwell exciton dispersion and, correspondingly, nonradiative character of the ground exciton state in parallel magnetic field. In high in-plane magnetic field the radiative decay rate becomes comparable and smaller than the nonradiative decay rate which results to the observed quenching of the interwell exciton PL intensity (Fig. 1a).

The unambiguous evidence for the nonradiative character of the ground state of interwell exciton in parallel magnetic field has been observed from the temperature dependence of the PL kinetics (Fig. 3). At zero field the exciton recombination rate monotonically reduces with increasing temperature (Fig. 3b) due to the thermal reduction of the radiative zone occupation [13]. In high in-plane magnetic field the temperature dependence is opposite: the exciton recombination rate enhances with increasing temperature (Fig. 3a) due to the increasing occupation of the radiative zone. Correspondingly, with increasing temperature the interwell exciton PL intensity reduces at zero field and enhances in high in-plane magnetic field (see insets to Fig. 3).

In conclusion, we have observed drastic change of the photoluminescence spectra and kinetics of interwell excitons in $\mathrm{GaAs} / \mathrm{Al}_{x} \mathrm{Ga}_{1-x} \mathrm{As} \mathrm{CQWs}$ in in-plane magnetic field. The effect is due to the in-plane magnetic field induced displacement of the indirect exciton dispersion in a momentum space, which results in the transition from the momentum-space direct exciton ground state to the momentum-space indirect exciton ground state. In-plane magnetic field is, therefore, an effective tool for the exciton dispersion engineering and controllable increase of the exciton ground state lifetime. We speculate that it can be used for the experimental realization of an ultra- low-temperature exciton gas, which might result in an observation of predicted collective phenomena caused by the high occupation of the lowest energy exciton states. In addition, the renormalization of the exciton mass due to in-plane magnetic field was observed; the experimental data are in a qualitative agreement with the theory.

We thank A. Imamoglu for discussions. We became aware on the studies of cw PL of interwell excitons in in-plane magnetic field [18]. We are grateful to the authors of Ref. [18] for providing us by their unpublished data and for discussions. We acknowledge support from INTAS, the Russian Foundation for Basic Research, and the Programme "Physics of Solid State Nanostructures" from the Russian Ministry of Sciences.

[1] Yu. E. Lozovik and V. I. Yudson, Zh. Eksp. Teor. Fiz. 71, 738 (1976) [Sov. Phys. JETP 44, 389 (1976)].

[2] Y. Kuramoto, and C. Horie, Solid State Commun. 25, 713 (1978).

[3] D. Yoshioka, and A.H. MacDonald, J. Phys. Soc. Jpn. 59, 4211 (1990).

[4] X.M. Chen, and J.J. Quinn, Phys. Rev. Lett. 67, 895 (1991).

[5] X. Zhu, P.B. Littlewood, M.S. Hybersten, and T.M. Rice, Phys. Rev. Lett. 74, 1633 (1995).

[6] L.P. Gor'kov and I.E. Dzyaloshinskii, Zh. Exp. Teor. Fiz. 53, 717 (1967) [Sov. Phys. JETP. 26, 449 (1968)].

[7] A.A. Gorbatsevich and I.V. Tokatly, Semicond. Sci. Technol. 13, 288 (1998).

[8] A.B. Dzyubenko and Yu.E. Lozovik, Fiz. Tverd. Tela 26, 1540 (1984) [Sov. Phys. Solid State 26, 938 (1984)].

[9] D. Paquet, T.M. Rice, and K. Ueda, Phys. Rev. B 32, 5208 (1985).

[10] A. Imamoglu, Phys. Rev. B 54, R14285 (1996).

[11] F. Stern, Phys. Rev. Lett. 21, 1687 (1968).

[12] L.V. Butov, A.A. Shashkin, V.T. Dolgopolov, K.L. Campman, and A.C. Gossard, Phys. Rev. B 60, 8753 (1999).

[13] L.V. Butov, A. Imamoglu, A.V. Mintsev, K.L. Campman, and A.C. Gossard, Phys. Rev. B 59, 1625 (1999).

[14] L.V. Butov, A. Imamoglu, K.L. Campman, and A.C. Gossard, unpublished.

[15] J. Feldmann, G. Peter, E.O. Göbel, P. Dawson, K. Moore, C. Foxon, and R.J. Elliott, Phys. Rev. Lett. 59, 2337 (1987).

[16] G.E.W. Bauer and T. Ando, Phys. Rev. B 38, 6015 (1988); B. Rejaei Salmassi and G.E.W. Bauer, ibid 39, 1970 (1989).

[17] D.M. Whittaker, T.A. Fisher, P.E. Simmonds, M.S. Skolnick, and R.S. Smith, Phys. Rev. Lett. 67, 887 (1991).

[18] A. Parlangeli, P.C.M. Christianen, J.C. Maan, I.V. Tokatly, C.B. Soerensen, and P.E. Lindelof, unpublished. 
FIG. 1. In-plane magnetic field dependence of the time integrated PL spectrum (a) and the interwell exciton PL kinetics (b) in the indirect regime $\left(V_{g}=1 \mathrm{~V}\right)$ at $T_{\text {bath }}=1.5 \mathrm{~K}$. Upper inset: The interwell exciton energy vs in-plane magnetic field, the line is the fitting curve for small fields $E_{p=0}=e^{2} d^{2} B^{2} / 2 M c^{2}$, see text. Lower inset: The fastest interwell exciton PL decay rate and the interwell exciton PL intensity enhancement after excitation switch off, $\Delta=\ln \left(I_{P L-\max } / I_{P L-p u l s e-e n d}\right)$, vs in-plane magnetic field.

FIG. 2. Schematic band diagram of the GaAs $/ \mathrm{Al}_{x} \mathrm{Ga}_{1-x} \mathrm{As}$ CQW (a). Scheme of the interwell exciton dispersion at zero (b) and finite (c) in-plane magnetic field; the photon dispersion as well as the radiative zone marked bold are also shown.

FIG. 3. The interwell exciton PL kinetics vs temperature in in-plane magnetic field $B=10 \mathrm{~T}$ (a) and at $B=0$ (b). Insets: The corresponding time integrated PL spectra. 


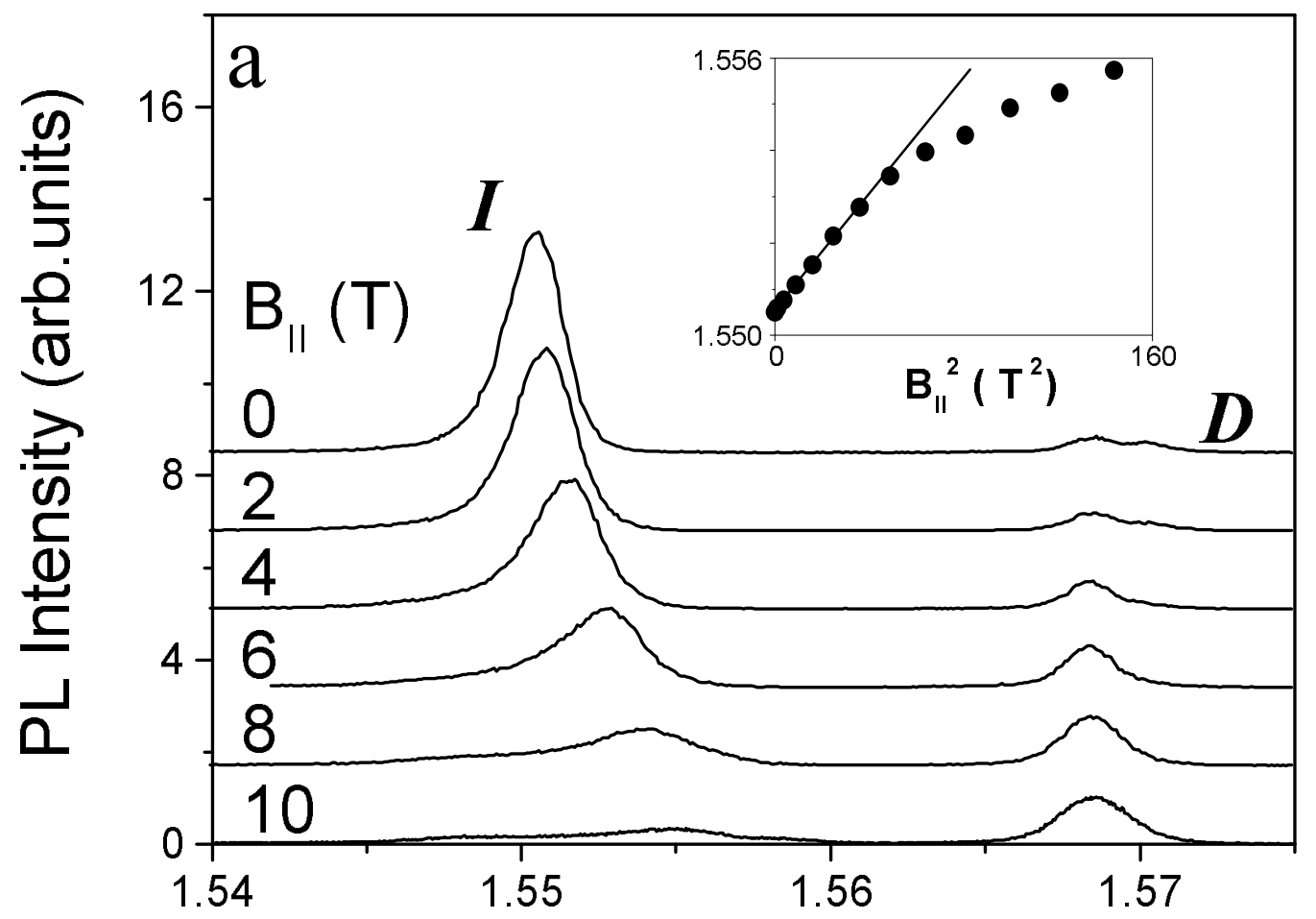

Energy (eV)

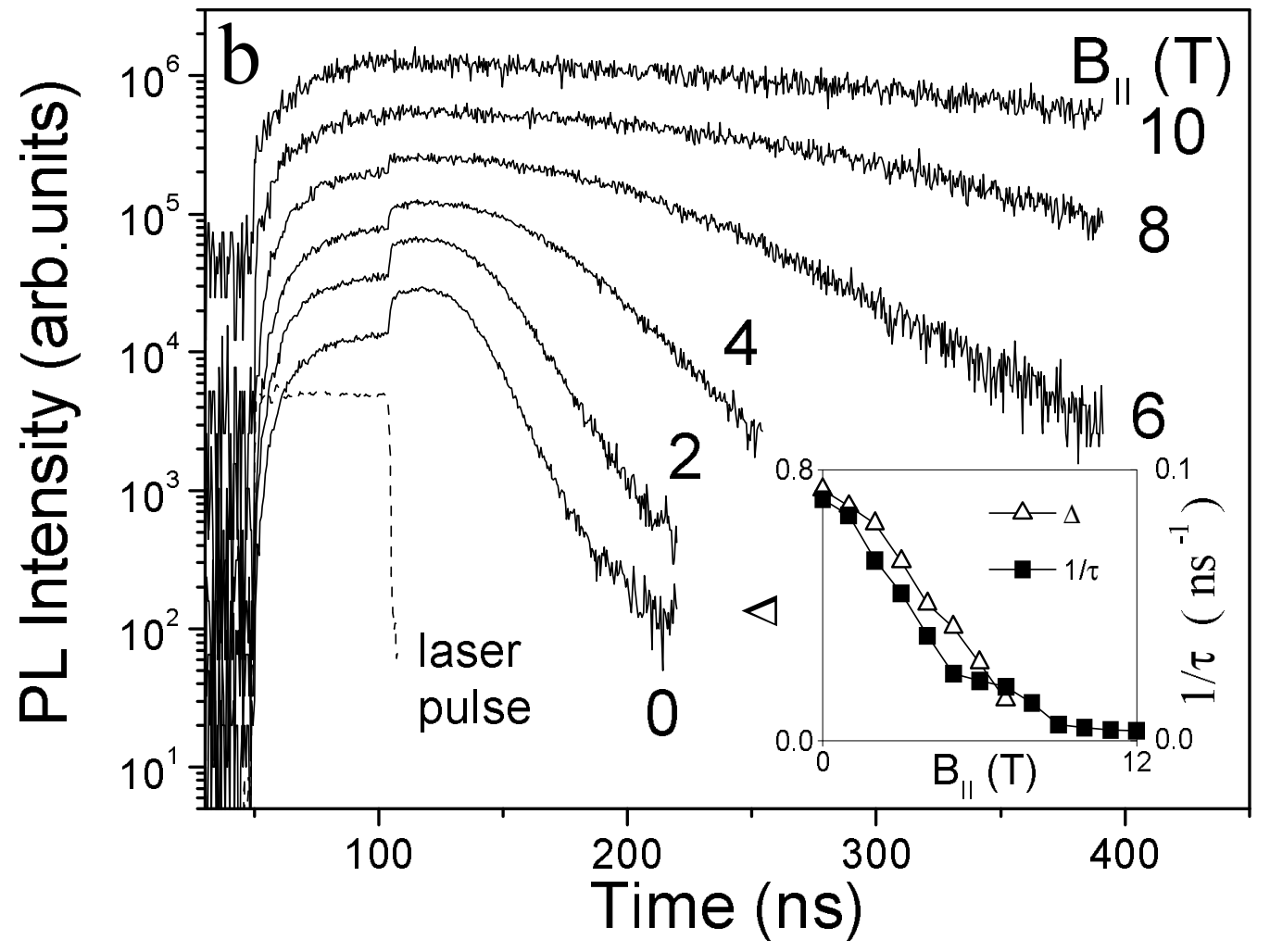




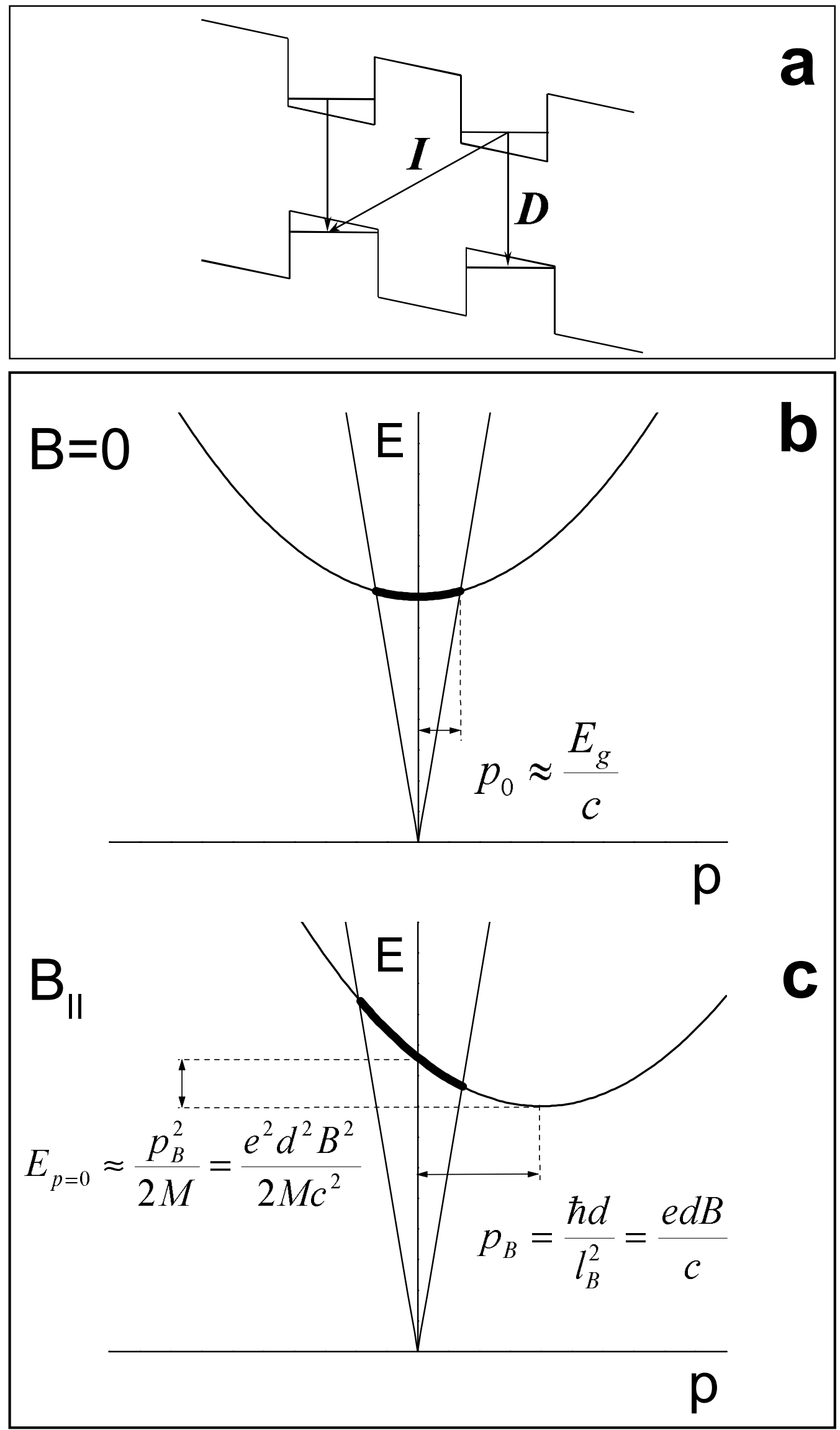



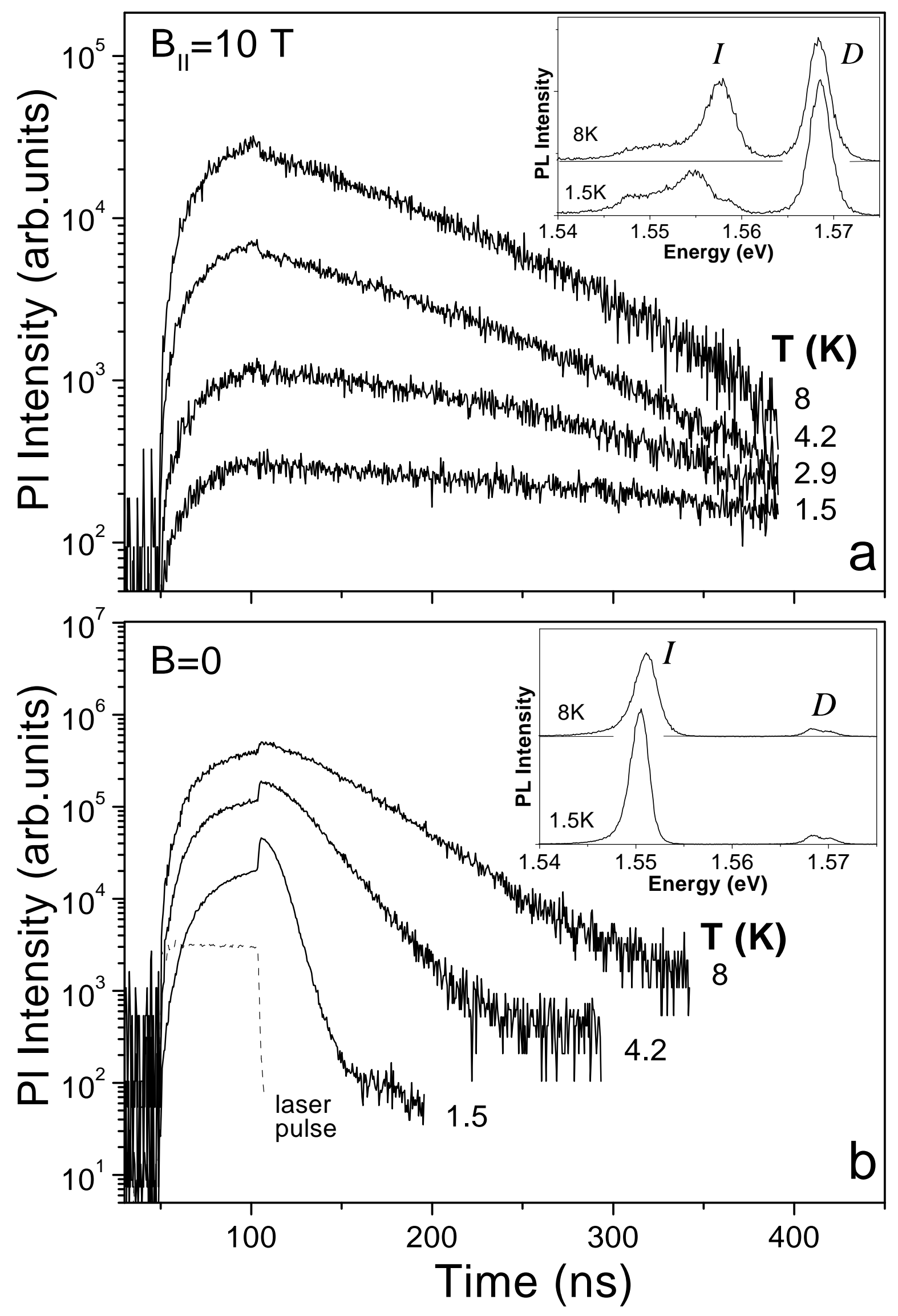